\title{
A Robust LCMP Beamformer with Limited Snapshots
}

\author{
Moaaz Mahdi $\quad$ Tarig Ballal ${ }^{\star \star} \quad$ Mohammad Moinuddin ${ }^{\star} \quad$ Tareq Y. Al-Naffouri ${ }^{\star \star} \quad$ Ubaid Al-Saggaf $^{\star}$ \\ * Department of Electrical and Computer Engineering, King Abdulaziz University, Jeddah, KSA \\ ** CEMSE, King Abdullah University of Science and Technology (KAUST), Thuwal, KSA
}

\begin{abstract}
This paper deals with the problem of automatic diagonal loading for the linear constrained minimum power (LCMP) beamformer. To find the beamformer's weights, the LCMP problem is reformulated into its generalized sidelobe canceller implementation, which is an unconstrained least squares (LS) problem. To solve this problem, we utilize a bounded perturbation regularization (BPR) approach where a perturbation matrix with a bounded norm is added to the linear transformation matrix of the LS problem in order to enhance the singular-value structure of the matrix. Compared to different diagonal loading methods, the proposed method shows superiority in performance when the the number snapshots is limited.
\end{abstract}

Index Terms-Robust adaptive beamforming, LCMP, generalized sidelobe canceller, diagonal loading.

\section{INTRODUCTION}

Beamforming is a spatial filtering technique that allows receiving desired signals impinging on an array from specific directions while suppressing undesired signals impinging from other directions [1].

Linearly constrained minimum variance (LCMP) beamforming minimizes the total power of the beamformer output such that a set of linear constraints that control the array beampattern is satisfied [2]. LCMP provides robustness against angle mismatch and perturbation in sensor locations [3]. Practically, the receiver does not have accurate spatial characteristic of a specific scenario. This makes filter designing methods base on assumptions that might not correspond completely to the actual parameters. Several reasons may attribute to this mismatch which include nonstationarity of the environment, multipath, small sample size, steering vector errors, etc. As a result, in the general case, performance of beamforemers deteriorate; hence, robust adaptive beamforming (RAB) techniques that mitigate the effect of such mismatches are required [4]. In the literature, a variety of RAB techniques were proposed.

Interference-plus-noise covariance (INC) matrix reconstruction methods aim at reducing the effect of the signal of interest (SOI) by reconstructing the INC [5], [6]. However, the reconstruction process increases the computational complexity.

An alternative RAB technique is the uncertainty set based technique. Methods that involve this technique estimate the SOI's steering vector by specifying a spherical uncertainty constraint on the steering vector [7]. However, the performance of these methods is limited to low signal to noise ratio (SNR).
In addition, these methods are computationally inefficient since they require solving second-order cone programming problems [6].

Steering vector projection (SVP) is another variation of RAB techniques [8], [9]. The steering vector is replaced by its projection on the signal-plus-interference subspace of the sample covariance matrix, which reduces the effect of noise disturbance. SVP methods perform poorly at low SNRs. Also, they require perfect knowledge of the dimension of the signalplus-interference subspace.

Diagonal loading (DL) is a widely used RAB technique in which a constant diagonal matrix is added to the sample covariance matrix. This technique is also known as regularization in the statistical literature [3]. DL's performance depends on the choice of a scalar loading parameter. Choosing the optimal DL automatically is a challenging problem [4]. There is no rigorous way of selecting the parameter since it depends on the noise level or it is based on a norm constraint of the weight vector [10]. A few methods were proposed to tackle the problem of automatically choosing the DL parameter. DL methods are efficient if the exact steering vetctors of the signal of interest and interference signals are known or small mismatches are exist.

The general linear combination-based (GLC) method [10] estimates the covariance matrix via a shrinkage method. Its estimation is based on the minimum mean square errors (MMSE) criterion. However, GLC's perfromramce degrades when the number of sensors is relatively large [11]. A recent covariance matrix estimation technique for data sampled from elliptical symmetric distribution is proposed in [12]. It is also based on the estimation of the optimal shrinkage parameters that minimizes the mean squared error.

The work in [13] considers computing diagonal loading automatically using a method proposed by Horel, Kennard and Baldwin (HKB) [14], which computes diagonal loading from the regularized least squares formulation. However, its performance degrades when the number of snapshots is relatively large [11].

In this paper, we propose a robust LCMP beamformer based on the bounded perturbation regularization approach [15], [16]. To deal with the constraints in the LCMP beamforming problem, we used the generalized sidlelobe canceller of LCMP 
that reformulates the problem to an unconstrained least squares problem. The estimated sample covariance matrix which is included in the linear transformation matrix of the LS problem is normally ill-conditioned which makes using regularization approach desirable. The regularization parameter is computed using a procedure that combines a constrained equation with a mean squared error criterion. This allows for automatic adjustment of regularization parameter required by the proposed robust beamformer.

\section{BACKGROUND}

\section{A. Signal model and LCMP beamformer}

We assume $D$ narrowband far-field signals impinging on an array of $N$ elements $(N>D)$. The $n$th snapshot of signals received by an array is given by [11]

$$
\mathbf{x}(n)=\sum_{i=0}^{D-1} \mathbf{a}_{i} s_{i}(n)+\mathbf{v}(n),
$$

where $\mathbf{x}(n) \in \mathbb{C}^{N}, \mathbf{a}_{i}$ denotes the steering vector associated with signal $i$, the subscript $i$ denotes a set of narrowband signals with $i=0$ (for the desired signal), $i=1,2 \cdots, D-1$ (for interference signals), and $\mathbf{v}(n) \in \mathbb{C}^{N}$ is a vector of Gaussian noise samples.

The output of a narrowband beamformer is obtained by multiplying the signal $\mathbf{x}(n)$ with a complex weight $\mathbf{w}$ and summing the result to obtain [17]

$$
y(n)=\mathbf{w}^{H} \mathbf{x}(n) .
$$

For LCMP, these weights are selected to minimize the output power of the beamformer as follows [17]:

$$
\mathbf{w}_{\text {opt }}=\underset{\mathbf{w}}{\operatorname{argmin}} \mathbf{w}^{H} \mathbf{R}_{\mathbf{x}} \mathbf{w} \quad \text { s.t. } \quad \mathbf{C}^{H} \mathbf{w}=\mathbf{g},
$$

where $\mathbf{R}_{\mathbf{x}}=\mathbb{E}\left[\mathbf{x}(n) \mathbf{x}^{H}(n)\right]$ is the data covariance matrix, $\mathbf{C} \in \mathbb{C}^{N \times P}$ is the constraint matrix, and $\mathbf{g}$ is a constraint vector with $P$ elements. The optimum solution is given by

$$
\mathbf{w}_{\mathrm{opt}}=\mathbf{R}_{\mathbf{x}}{ }^{-1} \mathbf{C}\left(\mathbf{C}^{H} \mathbf{R}_{\mathbf{x}}{ }^{-1} \mathbf{C}\right)^{-1} \mathbf{g} .
$$

Practically, the true covariance matrix is unknown; thus, it is usually replaced by the sample covariance matrix

$$
\widehat{\mathbf{R}}_{\mathbf{x}}=\frac{1}{K} \sum_{n=1}^{K} \mathbf{x}(n) \mathbf{x}^{H}(n),
$$

where $K$ is the number of snapshots. The estimated weights of the LCMP beamformer using (5) are given by

$$
\mathbf{w}_{\text {lcmp }}=\widehat{\mathbf{R}}_{\mathbf{x}}^{-1} \mathbf{C}\left(\mathbf{C}^{H} \widehat{\mathbf{R}}_{\mathbf{x}}^{-1} \mathbf{C}\right)^{-1} \mathbf{g} .
$$

\section{B. Generalized sidelobe canceler}

The optimization (3) can be formulated differently by decomposing $\mathbf{w}$ into two components: The first one in the constraint subspace, and the second one orthogonal to first [17], i.e.,

$$
\mathbf{w}=\mathbf{w}_{q}-\mathbf{B w}_{a}
$$

where $\mathbf{w}_{q} \in \mathbf{C}^{N}$ is a fixed quiescent weight vector calculated as

$$
\mathbf{w}_{q}=\mathbf{C}\left(\mathbf{C}^{H} \mathbf{C}\right)^{-1} \mathbf{g},
$$

and $\mathbf{B} \in \mathbb{C}^{N \times(N-P)}$ is a blocking matrix which is orthogonal to $\mathbf{C}\left(\mathbf{B}^{H} \mathbf{C}=[\mathbf{0}]_{\boldsymbol{N}-\boldsymbol{P} \times \boldsymbol{P}}\right)$. The blocking matrix is not unique. It is chosen such that $\mathbf{B}^{H} \mathbf{B}=\mathbf{I}$ which can be calculated as the eigenvectors corresponding to the $N-P$ non-zero eigenvalues of $\mathbf{I}-\mathbf{C}\left(\mathbf{C}^{H} \mathbf{C}\right)^{-1} \mathbf{C}^{H}$.

By substituting (7) in (3) and replacing $\mathbf{R}_{\mathbf{x}}$ with $\widehat{\mathbf{R}}_{\mathbf{x}}$, the problem can be reformulated as the following unconstrained LS:

$$
\min _{\mathbf{w}_{a}}\left(\mathbf{B} \mathbf{w}_{a}-\mathbf{w}_{q}\right)^{H} \widehat{\mathbf{R}}_{\mathbf{x}}\left(\mathbf{B} \mathbf{w}_{a}-\mathbf{w}_{q}\right)
$$

or

$$
\min _{\mathbf{w}_{a}}\left\|\mathbf{A} \mathbf{w}_{a}-\mathbf{b}\right\|^{2}
$$

where $\mathbf{A} \triangleq \widehat{\mathbf{R}}_{\mathbf{x}}^{\frac{1}{2}} \mathbf{B} \in \mathbb{C}^{N \times N-P}$ and $\mathbf{b} \triangleq \widehat{\mathbf{R}}_{\mathbf{x}}^{\frac{1}{2}} \mathbf{w}_{q}$. The above minimization corresponds to the following linear regression model:

$$
\mathbf{b}=\mathbf{A} \mathbf{w}_{a}+\mathbf{z},
$$

where $\mathbf{z} \in \mathbb{C}^{N}$ is a residual error vector. Since $\widehat{\mathbf{R}}_{\mathbf{x}}^{\frac{1}{2}}$ is normally ill-conditioned, and $\mathbf{b}$ is noisy, the application of regularization to estimate $\mathbf{w}_{a}$ is desirable. The regularized least square problem is stated as follows:

$$
\min _{\mathbf{w}_{a}}\left\|\mathbf{A} \mathbf{w}_{a}-\mathbf{b}\right\|^{2}+\gamma\left\|\mathbf{w}_{a}\right\|^{2}
$$

which has the closed-form solution

$$
\hat{\mathbf{w}}_{a}=\left(\mathbf{A}^{H} \mathbf{A}+\gamma \mathbf{I}\right)^{-1} \mathbf{A}^{H} \mathbf{b} \text {. }
$$

Remark: As can be seen from (13), we consider regularizing $\mathbf{A}^{H} \mathbf{A}$, which is of dimension $(N-P) \times(N-P)$ instead of $\widehat{\mathbf{R}}_{\mathbf{x}}$ which is of an $N \times N$ dimension. Hence, the inversion (13) is valid for fewer snapshots.

\section{The MSE of the RLS estimator}

The mean-squared error criterion (MSE) for the RLS is defined as

$$
\mathrm{MSE}=\operatorname{tr}\left[\mathbb{E}\left[\left(\hat{\mathbf{w}}_{a}-\mathbf{w}_{a}\right)\left(\hat{\mathbf{w}}_{a}-\mathbf{w}_{a}\right)^{H}\right]\right],
$$

where $\operatorname{tr}$ (.) denotes the matrix trace. The singular value decomposition (SVD) of $\mathbf{A}$ is

$$
\mathbf{A}=\mathbf{U} \boldsymbol{\Sigma} \mathbf{V}^{H}
$$

where $\mathbf{U} \in \mathbb{C}^{N \times N}, \boldsymbol{\Sigma}=\operatorname{diag}\left[\sigma_{1}, \sigma_{2}, \cdots \sigma_{N}\right]^{T}$, with $\sigma_{1}>$ $\sigma_{2}>\cdots>\sigma_{N}$, and $\mathbf{V} \in \mathbb{C}^{N-P \times N}$.

Substituting Equation (15) in (11), substituting the result in (13), and substituting the final result in (14) and manipulating, we obtain

$$
\begin{aligned}
\operatorname{MSE}=\sigma_{\mathrm{z}}^{2} \operatorname{tr}\left[\boldsymbol{\Sigma}^{2}\right. & \left.\left(\boldsymbol{\Sigma}^{2}+\gamma \mathbf{I}\right)^{-2}\right] \\
& +\gamma^{2} \operatorname{tr}\left[\left(\boldsymbol{\Sigma}^{2}+\gamma \mathbf{I}\right)^{-2} \mathbf{V}^{H} \mathbf{R}_{\mathbf{w}_{a}} \mathbf{V}\right],
\end{aligned}
$$


where $\mathbf{R}_{\mathbf{w}_{a}} \triangleq \mathbb{E}\left[\mathbf{w}_{a} \mathbf{w}_{a}^{H}\right]$. The derivative of (16) with respect to $\gamma$ can be taken and a critical point can be obtained by solving

$$
\begin{aligned}
\operatorname{MSE}^{\prime}= & -2 \sigma_{\mathrm{z}}^{2} \operatorname{tr}\left[\boldsymbol{\Sigma}^{2}\left(\boldsymbol{\Sigma}^{2}+\gamma \mathbf{I}\right)^{-3}\right] \\
& +2 \gamma \operatorname{tr}\left[\boldsymbol{\Sigma}^{2}\left(\boldsymbol{\Sigma}^{2}+\gamma \mathbf{I}\right)^{-3} \mathbf{V}^{H} \mathbf{R}_{\mathbf{w}_{a}} \mathbf{V}\right]=0
\end{aligned}
$$

However, a closed-form solution cannot be produced from Equation (17). An approximated formula can be written as follows (Equation (5) in [18]):

$$
\begin{aligned}
\operatorname{MSE}^{\prime} \approx & -2 \sigma_{\mathrm{z}}^{2} \operatorname{tr}\left[\boldsymbol{\Sigma}^{2}\left(\boldsymbol{\Sigma}^{2}+\gamma \mathbf{I}\right)^{-3}\right] \\
& +2 \gamma \frac{\operatorname{tr}\left(\mathbf{R}_{\mathbf{w}_{a}}\right)}{N} \operatorname{tr}\left[\boldsymbol{\Sigma}^{2}\left(\boldsymbol{\Sigma}^{2}+\gamma \mathbf{I}\right)^{-3}\right]=0 .
\end{aligned}
$$

Solving for $\gamma$, we can obtain the approximate minimizer of the MSE in (16). That is

$$
\gamma_{o} \approx \frac{N \sigma_{\mathrm{z}}}{\operatorname{tr}\left(\mathbf{R}_{\mathbf{w}_{a}}\right)}
$$

However, optimal $\gamma$ depends on unknown statistics of the signal. The following section describes our method to obtain $\gamma$ directly from the observed signals.

\section{THE BOUNDED PERTURBATION REGULARIZATION APPROACH}

The proposed model that is used for estimating a vector quantity $\mathbf{w}_{\mathbf{a}} \in \mathbb{C}^{N}$ is

$$
\mathbf{b} \approx(\mathbf{A}+\Delta) \mathbf{w}_{a}+\mathbf{z},
$$

where $\Delta \in \mathbb{C}^{N \times N-P}$ is a perturbation matrix. The aim of this perturbation is to modify the singular values of $\mathbf{A}$ in a way that helps improve the estimation of the vector $\mathbf{w}_{a}$. The perturbation matrix $\Delta$ is chosen such that:

$$
\|\Delta\|_{2} \leq \zeta
$$

where $\zeta$ is an unknown constant. The problem formulation for estimating $\mathbf{w}_{a}$ can be written as a min-max optimization problem

$$
\begin{gathered}
\min _{\hat{\mathbf{w}}_{\mathbf{a}}} \max _{\Delta}\left\|\mathbf{b}-(\mathbf{A}+\Delta) \hat{\mathbf{w}}_{a}\right\|_{2} \\
\text { subject to }\|\Delta\|_{2} \leq \zeta,
\end{gathered}
$$

that is, we seek an estimate of $\mathbf{w}_{a}$ that minimizes the maximum residual error over all possible bounded perturbations $\Delta$.

Using Minkowski's inequality [19] and manipulating, the problem can be reformulated as the following equivalent minimization problem [15]:

$$
\min _{\hat{\mathbf{w}}_{a}}\left\|\mathbf{b}-\mathbf{A} \hat{\mathbf{w}}_{a}\right\|_{2}+\zeta\left\|\hat{\mathbf{w}}_{a}\right\|_{2}
$$

which has a solution given by (13) with the following constraint:

$$
\gamma=\frac{\zeta\left\|\mathbf{b}-\mathbf{A} \hat{\mathbf{w}}_{a}\right\|_{2}}{\left\|\hat{\mathbf{w}}_{a}\right\|_{2}}
$$

where $\gamma$ is the unknown regularization parameter. Substituting (24) in (13) and manipulating, we obtain the following equation:

$$
\begin{aligned}
f_{1}(\gamma) & =\gamma^{2} \mathbf{b}^{H} \mathbf{A}\left(\mathbf{A}^{H} \mathbf{A}+\gamma \mathbf{I}\right)^{-2} \mathbf{A}^{H} \mathbf{b} \\
& -\zeta^{2}\left[\mathbf{b}^{H} \mathbf{b}-\mathbf{b}^{H} \mathbf{A}\left(\mathbf{A}^{H} \mathbf{A}+\gamma \mathbf{I}\right)^{-1} \mathbf{A}^{H} \mathbf{b}\right. \\
& \left.-\gamma \mathbf{b}^{H} \mathbf{A}\left(\mathbf{A}^{H} \mathbf{A}+\gamma \mathbf{I}\right)^{-2} \mathbf{A}^{H} \mathbf{b}\right]=0 .
\end{aligned}
$$

Applying the SVD (25) and manipulating we obtain

$$
f_{2}(\gamma)=\mathbf{b}^{H} \mathbf{U}\left(\boldsymbol{\Sigma}^{2}-\zeta^{2} \mathbf{I}\right)\left(\boldsymbol{\Sigma}^{2}+\gamma \mathbf{I}\right)^{-2} \mathbf{U}^{H} \mathbf{b}=0 .
$$

Equation (26) can be used to find $\gamma$. However, it requires knowing the value of $\zeta$. To remove dependency on $\zeta$, we seek an optimal value, $\zeta_{o}$, that satisfies (26) on average, i.e.,

$$
\mathbb{E}\left[\mathbf{b}^{H} \mathbf{U}\left(\boldsymbol{\Sigma}^{2}-\tilde{\zeta}_{o}^{2} \mathbf{I}\right)\left(\boldsymbol{\Sigma}^{2}+\tilde{\gamma}_{o} \mathbf{I}\right)^{-2} \mathbf{U}^{H} \mathbf{b}\right]=0 .
$$

Solving for $\zeta_{o}^{2}$ and manipulating, we obtain

$$
\zeta_{o}^{2}=\frac{\operatorname{tr}\left[\boldsymbol{\Sigma}^{2}\left(\boldsymbol{\Sigma}^{2}+\gamma_{o} \mathbf{I}\right)^{-2} \mathbf{U}^{H} \mathbf{R}_{\mathrm{b}} \mathbf{U}\right]}{\operatorname{tr}\left[\left(\boldsymbol{\Sigma}^{2}+\gamma_{o} \mathbf{I}\right)^{-2} \mathbf{U}^{H} \mathbf{R}_{\mathrm{b}} \mathbf{U}\right]},
$$

where $\mathbf{R}_{\mathrm{b}}=\mathbb{E}\left[\mathbf{b} \mathbf{b}^{H}\right]=\mathbf{U} \boldsymbol{\Sigma} \mathbf{V}^{H} \mathbf{R}_{\mathbf{w}_{a}} \mathbf{V} \boldsymbol{\Sigma} \mathbf{U}^{H}+\sigma_{\mathrm{z}}^{2} \mathbf{I}_{n}$. Substituting for $\mathbf{R}_{\mathrm{b}}$ in (28) yields

$$
\zeta_{o}^{2}=\frac{\sigma_{z}^{2} \operatorname{tr}\left[\boldsymbol{\Sigma}^{2}\left(\boldsymbol{\Sigma}^{2}+\gamma_{o} \mathbf{I}\right)^{-2}\right]+\operatorname{tr}\left[\boldsymbol{\Sigma}^{4}\left(\boldsymbol{\Sigma}^{2}+\gamma_{o} \mathbf{I}\right)^{-2} \mathbf{V}^{H} \mathbf{R}_{w_{a}} \mathbf{V}\right]}{\sigma_{z}^{2} \operatorname{tr}\left[\boldsymbol{\Sigma}^{2}\left(\boldsymbol{\Sigma}^{2}+\gamma_{o} \mathbf{I}\right)^{-2}\right]+\operatorname{tr}\left[\boldsymbol{\Sigma}^{2}\left(\boldsymbol{\Sigma}^{2}+\gamma_{o} \mathbf{I}\right)^{-2} \mathbf{V}^{H} \mathbf{R}_{w_{a}} \mathbf{V}\right]}
$$

Applying a similar approximation to that used in (18), we reach

$$
\zeta_{o}{ }^{2} \approx \frac{\sigma_{z}^{2} \operatorname{tr}\left[\boldsymbol{\Sigma}^{2}\left(\boldsymbol{\Sigma}^{2}+\gamma_{o} \mathbf{I}\right)^{-2}\right]+\frac{\operatorname{tr}\left(\mathbf{R}_{w_{a}}\right)}{N} \operatorname{tr}\left[\boldsymbol{\Sigma}^{4}\left(\boldsymbol{\Sigma}^{2}+\gamma_{o} \mathbf{I}\right)^{-2}\right]}{\sigma_{z}^{2} \operatorname{tr}\left[\boldsymbol{\Sigma}^{2}\left(\boldsymbol{\Sigma}^{2}+\gamma_{o} \mathbf{I}\right)^{-2}\right]+\frac{\operatorname{tr}\left(\mathbf{R}_{w_{a}}\right)}{N} \operatorname{tr}\left[\boldsymbol{\Sigma}^{2}\left(\boldsymbol{\Sigma}^{2}+\gamma_{o} \mathbf{I}\right)^{-2}\right]},
$$

which can be written as

$$
\zeta_{o}^{2}=\frac{\operatorname{tr}\left[\boldsymbol{\Sigma}^{2}\left(\boldsymbol{\Sigma}^{2}+\gamma_{o} \mathbf{I}\right)^{-1}\right]}{\operatorname{tr}\left[\left(\boldsymbol{\Sigma}^{2}+\gamma_{o} \mathbf{I}\right)^{-1}\right]} .
$$

Finally, substituting (31) in (26) and replacing $\gamma_{o}$ with $\gamma$ and manipulating yields

$$
\begin{aligned}
f_{3}(\gamma) & =\operatorname{tr}\left[\left(\boldsymbol{\Sigma}^{2}+\gamma \mathbf{I}\right)^{-1}\right] \operatorname{tr}\left[\left(\boldsymbol{\Sigma}^{2}+\gamma \mathbf{I}\right)^{-1} \mathbf{d d}^{H}\right] \\
& -N\left[\left(\boldsymbol{\Sigma}^{2}+\gamma \mathbf{I}\right)^{-2} \mathbf{d d}^{H}\right]=0,
\end{aligned}
$$

where $\mathbf{d} \triangleq \mathbf{U}^{H} \mathbf{b}$. Equation (32) is the BPR equation which can be solved using Newton's or any other suitable method [15].

Finally, we substitute the regularization parameter, $\gamma$, obtained from (32) in the loaded version of (6), to obtain the following:

$$
\mathbf{w}_{\mathrm{DL}}=\left(\widehat{\mathbf{R}}_{\mathbf{x}}+\gamma \mathbf{I}\right)^{-1} \mathbf{C}\left(\mathbf{C}^{H}\left(\widehat{\mathbf{R}}_{\mathbf{x}}+\gamma \mathbf{I}\right)^{-1} \mathbf{C}\right)^{-1} \mathbf{g} .
$$




\section{Simulation Results}

To evaluate performance, the output signal-to-interferenceand-noise-ratio (SINR) is considered. Equation (1) can be written as $\mathbf{x}(n)=\mathbf{x}_{s}(n)+\mathbf{x}_{i v}(n)$, where $\mathbf{x}_{s}(n) \triangleq \mathbf{a}_{0} s_{0}(n)$ and $\mathbf{x}_{i v}(n) \triangleq \sum_{i=1}^{D-1} \mathbf{a}_{i} s_{i}(n)+\mathbf{v}(n)$. The output SINR is defined as follows:

$$
\begin{aligned}
\mathrm{SINR} & =\frac{\mathbb{E}\left[\left|\mathbf{w}^{H} \mathbf{x}_{s}(n)\right|^{2}\right]}{\mathbb{E}\left[\left|\mathbf{w}^{H} \mathbf{x}_{i v}(n)\right|^{2}\right]} \\
& =\frac{\sigma_{s}^{2}\left|\mathbf{w}^{H} \mathbf{a}_{0 t}\right|^{2}}{\mathbf{w}^{H} \mathbf{R}_{i+n} \mathbf{w}},
\end{aligned}
$$

where $\mathbf{R}_{i+n}=\mathbb{E}\left[\mathbf{x}_{i v}(n) \mathbf{x}_{i v}^{H}(n)\right]$ is the interference-plus-noise covariance (INC) matrix, $\sigma_{s}^{2}$ is the power of the signal of interest, and $\mathbf{a}_{0 t}$ is the actual steering vector of the desired signal.

In all scenarios, we compare the proposed LCMP-BPR with HKB [14], [13], elliptical regularized sample covariance matrix (ELL-RSCM) [12], GLC [10], multichannel wiener filtering based noise reduction with truncated minimum mean square error criterion (MWF-TMMSE) [11], and tridiagonal loading (TRI) [20] methods. Similar to the proposed method, HKB and TRI methods are one parameter diagonal loading methods. The other methods, Ell-RSCP, GLC and MWFTMMSE, estimate the covariance matrix via a shrinkage method that uses two regularization parameters.

A uniform linear array (ULA) of $N=10$ elements with $d=0.5 \lambda$ between consecutive elements is used in all simulations, where $\lambda$ is the wavelength. Uncertainty in the direction of arrival (DOA) of the signal of interest is modeled as a uniform distribution in the range $\left[-2^{\circ}, 2^{\circ}\right]$. We consider six interference signals $(D=6)$ impinging on the array.

The signal of interest and interference signals are complex Gaussian data generated randomly with $\mathrm{SNR}=5 \mathrm{~dB}$ and interference-to-noise ratio (INR $=20 \mathrm{~dB}$ ). The noise is complex white Gaussian with unit-norm power. Source locations are randomly chosen in every iteration. All SINR curves are obtained by averaging over $2 \times 10^{4}$ independent trials. For this LCMP beamformer, three signals out of the six interference signals are constrained to nulls, i.e., the constraint vector is $\mathbf{g}=[1,0,0,0]^{T}(P=4)$.

Fig. 1 (a) shows the output SINR versus SNR. As can be seen from the figure, for SNR $\leq 10 \mathrm{~dB}$, the proposed LCMPBPR method achieves the best performance among all the methods. For $10 \mathrm{~dB}<\mathrm{SNR} \leq 20 \mathrm{~dB}$, LCMP-BPR exhibits an inferior performance compared to the other methods except HKB.

Fig. 1 (b) shows the output SINR versus the number of snapshots, $K$. As can be seen, when $N-P<K<2 N$, LCMP-BPR outperforms all the other techniques. However, for $2 N \leq K \leq 3 N$, HKB shows a slight advantage over the proposed LCMP-BPR.

It is worth mentioning that the performance of our method is highly sensitive to the effect of the desired signal in the estimated INC. This explains the good performance of our method at low SNRs. To elaborate, we consider estimating the
INC matrix from the noise and interference samples generated in the simulation, i.e.,

$$
\widehat{\mathbf{R}}_{i+n}=\frac{1}{K} \sum_{n=1}^{K} \mathbf{x}_{i v}(n) \mathbf{x}_{i v}^{H}(n),
$$

and use it instead of $\widehat{\mathbf{R}}_{\mathbf{x}}$. Fig. 2 (a) illustrates the output SINR versus SNR performance for INC estimated using (36) . It is clear that our method outperforms all the methods over the entire SNR range. Similarly, Fig. 2 (b) shows the performance of SINR versus the number of snapshots with enhanced estimation of INC. It is evident that both LCMP-BPR and HKB outperform the methods with a good margin. The superiority of BPR-LCMP is more emphasized for $N-P<K \leq 3 N$.

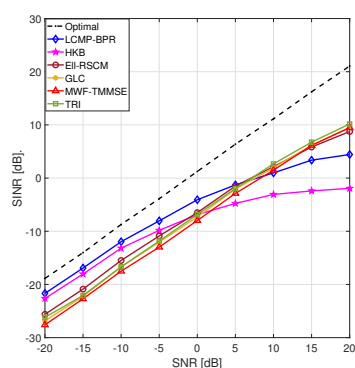

(a) SINR versus $\operatorname{SNR}(K=7)$

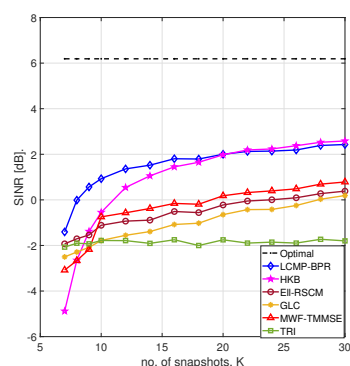

(b) SINR versus $K(\mathrm{SNR}=5 \mathrm{~dB})$
Fig. 1. Output SINR performance (good estimation of INC matrix).

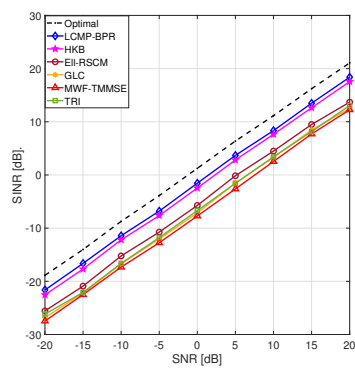

(a) $\mathrm{SINR}$ versus $\mathrm{SNR}(K=7)$

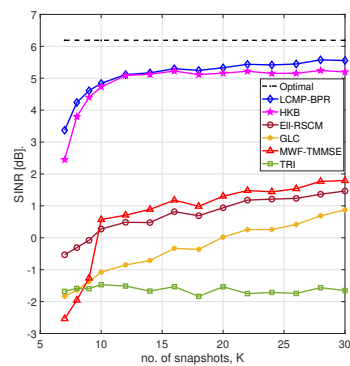

(b) SINR versus $K(\mathrm{SNR}=5 \mathrm{~dB})$
Fig. 2. Output SINR performance (better estimation of INC matrix ).

\section{CONCLUSION}

We propose the LCMP-BPR beamformer based on the bounded perturbation regularization approach. A generalized sidelobe canceller implementation of a linearly constrained minimum power was considered. The constrained LCMP problem was reformulated into an unconstrained least squares problem. Simulation results show that the proposed LCMPBPR method is effective in scenarios with a ULA of $N$ elements, $P$ constraints, and limited number of snapshot $K$ $\in(N-P, 3 N]$. 


\section{REFERENCES}

[1] B. D. Van Veen and K. M. Buckley, "Beamforming: a versatile approach to spatial filtering," IEEE ASSP Magazine, vol. 5, no. 2, pp. 4-24, April 1988.

[2] A. Hassani, A. Bertrand, and M. Moonen, "Lcmv beamforming with subspace projection for multi-speaker speech enhancement," in 2016 IEEE International Conference on Acoustics, Speech and Signal Processing (ICASSP). IEEE, 2016, pp. 91-95.

[3] H. L. Van Trees, Optimum array processing: Part IV of detection, estimation, and modulation theory. John Wiley \& Sons, 2004.

[4] J. Li and P. Stoica, Robust adaptive beamforming. Wiley Online Library, 2006.

[5] Y. Gu and A. Leshem, "Robust adaptive beamforming based on interference covariance matrix reconstruction and steering vector estimation," IEEE Transactions on Signal Processing, vol. 60, no. 7, pp. 3881-3885, 2012.

[6] Z. Zheng, Y. Zheng, W.-Q. Wang, and H. Zhang, "Covariance matrix reconstruction with interference steering vector and power estimation for robust adaptive beamforming," IEEE Transactions on Vehicular Technology, vol. 67, no. 9, pp. 8495-8503, 2018.

[7] A. Khabbazibasmenj, S. A. Vorobyov, and A. Hassanien, "Robust adaptive beamforming based on steering vector estimation with as little as possible prior information," IEEE Transactions on Signal Processing, vol. 60, no. 6, pp. 2974-2987, June 2012.

[8] D. D. Feldman and L. J. Griffiths, "A projection approach for robust adaptive beamforming," IEEE Transactions on signal processing, vol. 42, no. 4, pp. 867-876, 1994.

[9] L. Huang, B. Zhang, and Z. Ye, "Robust adaptive beamforming using a new projection approach," in 2015 IEEE International Conference on Digital Signal Processing (DSP), July 2015, pp. 1181-1185.

[10] L. Du, J. Li, and P. Stoica, "Fully automatic computation of diagonal loading levels for robust adaptive beamforming," IEEE Transactions on Aerospace and Electronic Systems, vol. 46, no. 1, pp. 449-458, 2010.

[11] Y. Ke, C. Zheng, R. Peng, and X. Li, "Robust adaptive beamforming using noise reduction preprocessing-based fully automatic diagonal loading and steering vector estimation," IEEE Access, vol. 5, pp. 12974 12987, 2017.

[12] E. Ollila and E. Raninen, "Optimal shrinkage covariance matrix estimation under random sampling from elliptical distributions," IEEE Transactions on Signal Processing, vol. 67, no. 10, pp. 2707-2719, 2019.

[13] Y. Selén, R. Abrahamsson, and P. Stoica, "Automatic robust adaptive beamforming via ridge regression," Signal Processing, vol. 88, no. 1, pp. 33-49, 2008.

[14] A. E. Hoerl, R. W. Kannard, and K. F. Baldwin, "Ridge regression: some simulations," Communications in Statistics-Theory and Methods, vol. 4, no. 2, pp. 105-123, 1975

[15] T. Ballal, M. A. Suliman, and T. Y. Al-Naffouri, "Bounded perturbation regularization for linear least squares estimation," IEEE Access, vol. 5, pp. 27 551-27 562, 2017.

[16] M. Suliman, T. Ballal, and T. Y. Al-Naffouri, "Robust regularized leastsquares beamforming approach to signal estimation," in 2016 IEEE Global Conference on Signal and Information Processing (GlobalSIP), Dec 2016, pp. 75-79.

[17] Z. Tian, K. L. Bell, and H. L. Van Trees, "A recursive least squares implementation for lcmp beamforming under quadratic constraint," IEEE Transactions on Signal Processing, vol. 49, no. 6, pp. 1138-1145, 2001.

[18] S.-D. Wang, T.-S. Kuo, and C.-F. Hsu, "Trace bounds on the solution of the algebraic matrix riccati and lyapunov equation," IEEE Transactions on Automatic Control, vol. 31, no. 7, pp. 654-656, 1986.

[19] L. Maligranda, "A simple proof of the hölder and the minkowski inequality," The American mathematical monthly, vol. 102, no. 3, pp. 256-259, 1995.

[20] M. Zhang, X. Chen, and A. Zhang, "A simple tridiagonal loading method for robust adaptive beamforming," Signal Processing, vol. 157, pp. 103 107, 2019. 